\title{
Evaluation of dyskerin expression and the Cajal body protein WRAP53 $\beta$ as potential prognostic markers for patients with primary vaginal carcinoma
}

\author{
CECILIA RANHEM ${ }^{1,2}$, GABRIELLA LILLSUNDE LARSSON $^{3,4}$, DAVID LINDQUIST $^{5}$, \\ BENGT SORBE $^{6}$, MATS G. KARLSSON ${ }^{7}$, MARIANNE FARNEBO $^{8,9}$, KRISTINA HELLMAN $^{10}$, \\ LARYSA KOVALESKA $^{11}$, ELENA KASHUBA ${ }^{11,12^{*}}$ and SONIA ANDERSSON ${ }^{1 *}$
}

\author{
${ }^{1}$ Department of Women's and Children's Health, Karolinska Institutet, 17177 Stockholm; \\ ${ }^{2}$ Centre for Clinical Research Region Västmanland, Uppsala University, Västmanlands Hospital Västerås, \\ 72189 Västerås; ${ }^{3}$ School of Health and Medical Sciences, Örebro University, Campus USÖ, 70182 Örebro; \\ ${ }^{4}$ Department of Laboratory Medicine, Örebro University Hospital, 70185 Örebro; ${ }^{5}$ Department of Radiation Sciences, \\ Umeå Universitet, 90187 Umeå; ${ }^{6}$ Department of Oncology, Örebro University Hospital; ${ }^{7}$ School of Medical Sciences, \\ Örebro University, 70185 Örebro; ${ }^{8}$ Department of Bioscience and Nutrition, Karolinska Institutet, 14183 Stockholm; \\ ${ }^{9}$ Department of Cell and Molecular Biology, Karolinska Institutet, 17177 Stockholm; \\ ${ }^{10}$ Department of Oncology-Pathology, Karolinska Institutet, 17164 Stockholm, Sweden; \\ ${ }^{11}$ R.E. Kavetsky Institute of Experimental Pathology, Oncology and Radiobiology of \\ National Academy of Sciences of Ukraine, 03022 Kiev, Ukraine; ${ }^{12}$ Department of Microbiology, \\ Tumour and Cell Biology, Karolinska Institutet, 17165 Stockholm, Sweden
}

Received April 1, 2021; Accepted September 30, 2021

DOI: $10.3892 / 01.2021 .13148$

\begin{abstract}
Primary vaginal cancer (PVC) is a rare gynaecological malignancy, which, at present, lacks appropriate biomarkers for prognosis. The proteins dyskerin and WD repeat containing antisense to TP53 (WRAP53 $\beta$ ), both of which exert their functions in the telomerase holoenzyme complex, have been shown to be upregulated in different cancer types. These proteins have also been proposed as prognostic markers in some types of cancer. The aim of the present study was to examine the expression patterns of dyskerin and WRAP53 $\beta$ in patients with PVC. Moreover, as part of a search for effective biomarkers to evaluate prognosis in PVC, the expression of these two proteins and their
\end{abstract}

Correspondence to: Dr Cecilia Ranhem, Department of Women's and Children's Health, Karolinska Institutet, Widerströmska Huset, Tomtebodavägen 18A, 17177 Stockholm, Sweden

E-mail: cecilia.ranhem@regionvastmanland.se

${ }^{*}$ Contributed equally

Abbreviations: PVC, primary vaginal carcinoma; TERT, telomerase reverse transcriptase; TERC, telomerase RNA complex; HPV, human papillomavirus; IHC, immunohistochemical; WRAP53 $\beta$, WD repeat containing antisense to TP53

Key words: primary vaginal carcinoma, dyskerin, Cajal body, WRAP53 $\beta$, biomarker, prognosis, survival potential association with clinical variables and survival were also evaluated. The expression of dyskerin and WRAP53 $\beta$ was assessed in PVC tumour samples from 68 patients using immunohistochemistry. The majority of tumour samples showed low and moderate expression levels of dyskerin. Upregulation of dyskerin in tumour samples was significantly associated with a shorter survival time and a poorer cancer-specific survival rate. WRAP53 $\beta$ was also expressed in most of the cells but was not significantly associated with clinical variables or survival. This study demonstrates that upregulation of dyskerin is significantly associated with poor prognosis. Thus, dyskerin may serve as a promising prognostic marker and a potential putative therapeutic target in PVC.

\section{Introduction}

Primary vaginal carcinoma (PVC) accounts for $1-2 \%$ of all gynaecological malignancies and mainly affects postmenopausal women, the majority of whom are diagnosed at an early stage (1-3). Radiation therapy, with or without concurrent chemotherapy, is considered to be the treatment of choice and results in 5-year cancer-specific survival rates of $\sim 70 \%$ (4). Due to its rarity, few studies have been conducted on PVC, and therefore knowledge concerning biological factors and biomarkers, both diagnostic and prognostic, is limited. Established prognostic factors include age, tumour size, and stage (5-8). Several molecular biomarkers have been proposed, including p16, Ki67, p53, EGFR, VEGF and human papillomavirus (HPV) infection, although findings regarding these markers have been inconsistent $(7,9,10)$. Therefore, 
identification of new biomarkers is essential for the improvement of diagnosis, treatment outcome and prognosis for patients with PVC.

Cancer cells are able to proliferate constitutively (11). One important mechanism that supports endless proliferation is the addition of telomere repeats, which protect chromosomes from shortening. This process is carried out by the telomerase enzyme, a ribonucleoprotein complex consisting of two components: The telomerase reverse transcriptase (TERT) and the telomerase RNA complex (TERC) (12). Additional factors required for the catalytical activity of telomerase include dyskerin (also known as dyskerin pseudouridine synthase 1) and the WD repeat containing antisense to TP53 $\beta$ (WRAP53 $\beta$ ) protein (also known as telomerase Cajal body protein 1 or as WD repeat domain 79) (13). Dyskerin serves as a backbone of the telomerase complex. WRAP53 $\beta$ is required for telomere synthesis in human cancer cells, as this protein localises telomerase to telomeres (14). Dyskerin and WRAP53 $\beta$ are usually localised in nuclear organelles known as Cajal bodies, where they are involved in both the biogenesis of telomerase and in spliceosomal machinery $(14,15)$.

Dyskerin, a pseudouridine synthase, is also found in the nucleoli of cells (16), where it is responsible for the modification of ribosomal RNA molecules important to ribosome biogenesis. When dysregulated, this protein has been associated with various cancer types, including breast cancer (17), renal cancer (18) pituitary tumours (19) and glioma (20). In addition, increased expression of dyskerin has often been associated with worse prognosis $(16,18,21-23)$. To our knowledge, dyskerin has not previously been studied in relation to gynaecological malignancies.

WRAP $53 \beta$, originally identified as an antisense gene to the TP53 tumour suppressor (24), is a scaffolding protein involved in the intracellular trafficking of RNA, telomerase and DNA repair proteins. WRAP $53 \beta$ has been linked to a variety of cellular processes, including the maintenance of Cajal bodies (24), telomere elongation (14) and DNA repair (25). Loss of WRAP53 $\beta$ is associated with poor prognosis in head and neck (26), breast (27) and ovarian cancer (28), suggesting a tumour suppressor role $(29,30)$. Although upregulation of WRAP53 $\beta$ has also been reported in cancer, correlation with patient survival has been inconsistent (31-33). Instead, this upregulation, which is known to occur in precancerous lesions, may reflect WRAP53 $\beta$ involvement in DNA repair in order to constrain tumour progression $(29,30)$.

The present study aimed to examine the expression patterns of dyskerin and WRAP53 $\beta$ in patients with PVC. Moreover, as part of a search for effective biomarkers to evaluate prognosis in PVC, the expression of these two proteins and their potential association with clinical variables and survival was also evaluated.

\section{Materials and methods}

Sample collection. The present study is based on archived diagnostic PVC tumour samples from a consecutive cohort of 81 women. The inclusion criteria were women diagnosed with and treated for PVC between January 1975 and December 2002 at Örebro University Hospital or at the central hospitals in Eskilstuna, Västerås, and Karlstad (9). Seven cases were excluded after immunohistochemical evaluation due to insufficient tumour samples.

The clinical characteristics of this cohort have been previously described in a study by Larsson et al (9), including information on age at diagnosis (mean, 69.4 years; range, 37-90 years), tumour size, International Federation of Gynecology and Obstetrics (FIGO) stage, tumour localisation, histological type (including basaloid squamous cell carcinoma, non-keratinising squamous cell carcinoma, keratinising squamous cell carcinoma, verrucous squamous cell carcinoma, adenocarcinoma, sarcoma and melanoma, based on World Health Organisation criteria) and tumour grade (34). Treatment and follow-up data for each patient were obtained through hospital records. All patient records were subjected to retrospective follow-up from the time of diagnosis. Median follow-up time for patients who were alive at the end of the study was 121 months (range, 44-290 months). As in the previous protocol by Larsson et al (9), complete remission was defined as disappearance of all clinical evidence of disease after primary treatment, while tumour recurrence was defined as detection of cancer after a period of at least 6 months following initial complete remission.

Information on HPV status was also reported in Larsson et al (9). Of the 81 tumour samples, 37 were HPV-positive, 34 were HPV-negative, and 10 had insufficient material for HPV detection. Of the 37 HPV-positive cases, 26 (70\%) were HPV16-positive, while the remaining 11 were positive for other high-risk HPV genotypes.

Immunohistochemical staining and analysis of dyskerin and WRAP53 $\beta$. In all, 68 of the 81 tumour samples were found to be appropriate for immunohistochemical staining and analysis of dyskerin and WRAP53 $\beta$. The paraffin-embedded tumour samples were cut into 5- $\mu \mathrm{m}$ sections and established to be representative by two pathologists (MGK and OG). Paraffin was then dissolved in xylene, and the tissue samples were rehydrated by stepwise washing with 96 and $70 \%$ ethanol in phosphate-buffered saline. The tissue samples were then immersed in a $2 \%$ solution of $\mathrm{H}_{2} \mathrm{O}_{2}$ in methanol at room temperature for $30 \mathrm{~min}$ to reduce background staining. Epitopes were retrieved by heating in citrate buffer (water bath, $96^{\circ} \mathrm{C}$ for $15 \mathrm{~min}$ ), and the tumour samples were then cooled to room temperature. The primary anti-dyskerin (cat. no. sc-373956; Santa Cruz Biotechnology, Inc.) and anti-WRAP53 (cat. no. PA-2020-100; Innovagen AB) antibodies, diluted 1:200 in a blocking buffer ( $2 \%$ bovine serum albumin, $0.2 \%$ Tween-20, $10 \%$ glycerol and $0.05 \% \mathrm{NaN}_{3}$ in phosphate-buffered saline; all from Sigma-Aldrich; Merck KGaA) were applied and left to stand for $60 \mathrm{~min}$ at room temperature. Protein signals were visualised using the secondary antibodies provided in the EnVision ${ }^{\mathrm{TM}}$ Detection Peroxidase/DAB system kit (Dako; Agilent Technologies, Inc.), which was used according to the manufacturer's protocol. Nuclei were stained with Mayer's haematoxylin (Dako; Agilent Technologies, Inc.).

The dyskerin nuclear signal was assessed as: i) Negative (no positive cells observed); ii) weak (1+); iii) moderate (2+); and iv) and strong (3+) by an experienced pathologist (OG), using a light microscope Leica (magnification, $\mathrm{x} 400$ ). Tumour cells were only analysed in sections in which the total number 

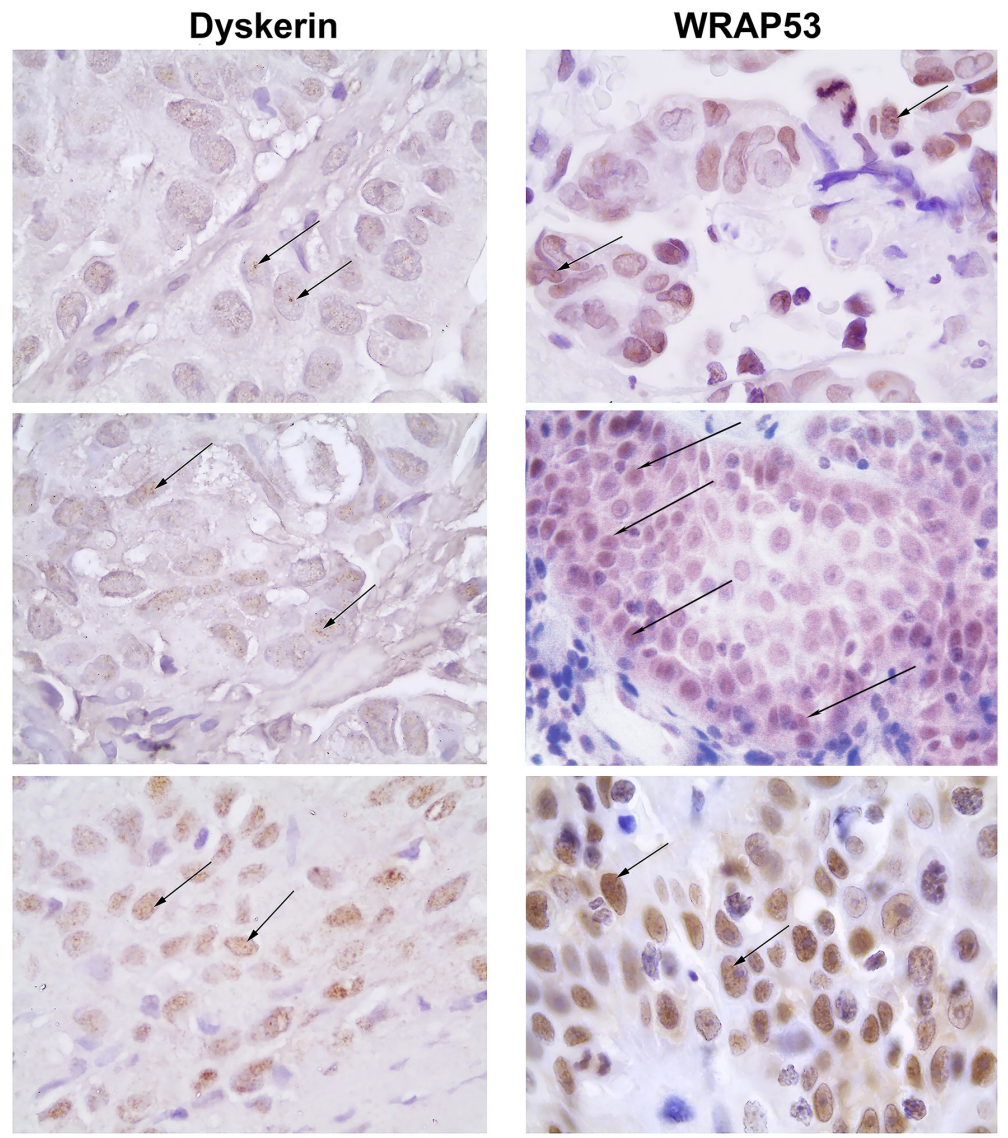

Figure 1. Expression pattern of dyskerin and WRAP53 $\beta$ in tumour samples of three representative patients with primary vaginal cancer (magnification, $x 400$ ). The expression of dyskerin and WRAP53 $\beta$ was assessed using immunohistochemistry (in brown). On the left column, dyskerin is indicated with arrows and located in the nucleoli (top panel), nuclear bodies (middle panel) and is dispersed in nucleoplasm (bottom panel). On the right column, arrows indicate presence of WRAP53 $\beta$ in nuclear bodies located in the nucleoplasm, i.e. not nucleoli. Nuclei are shown in blue. WRAP53 $\beta$, WD repeat containing antisense to TP53 $\beta$.

of cells was $\geq 400$ (minimum of 5 fields). The location of the protein signals detected within the nucleoplasm and/or in nuclear bodies was also recorded. In the present study, nuclear bodies refer to nucleoplasmic bodies, excluding nucleoli.

Two experienced pathologists (OG and MGK) carried out microscopic evaluation of WRAP53 $\beta$ signals based on the fraction of stained tumour cells and on staining intensity. The percentage of positive cells was categorised into four, semi-quantitative groups: i) 0 , negative; ii) $1,<25 \%$; iii) $2,25-50 \%$; and iv) $3,>50 \%$ of cells (MKG). In addition, staining intensity was graded as: i) 0 , negative (no positive cells observed); ii) 1 , weak; iii) 2 , moderate; and iv) 3 , strong.

Statistical analysis. Pearson's $\chi^{2}$ test or Fisher's exact tests were used to analyse the association between ordinal variables and IHC parameters. An independent-sample t-test was used to analyse the differences between the means of the groups. Survival analysis according to the expression of dyskerin and WRAP53 $\beta$ is presented as a Kaplan-Meier graph, and a log-rank test was used to compare the different dichotomised groups. Multivariate analysis of different prognostic factors, such as age at diagnosis, tumour size, histology and FIGO stage, was performed using the Cox proportional-hazards model for survival outcome. Statistical analysis was carried out using SPSS 19 software (IBM Corp.). $\mathrm{P}<0.05$ was considered to indicated a statistically significant difference.
Table I. IHC analysis of dyskerin staining intensity and localisation pattern in tumour samples of patients with primary vaginal cancer.

IHC parameter n $\quad \mathrm{n} \%)$

Dyskerin IHC staining intensity

Negative (no positive cells)

$1+($ weak)

$2+$ (moderate)

$3+$ (strong)

Dyskerin localisation

Nuclear bodies (negative nucleoplasm)

Nucleoplasm (negative nuclear bodies)

Nuclear bodies + nucleoplasm

No staining or very weak

$5(7.3)$

IHC, immunohistochemical.

\section{Results}

Immunohistochemical analysis of dyskerin. Immunohistochemical analysis of 68 tumour samples revealed varying degrees of nuclear expression of dyskerin (Fig. 1 and Table I). 


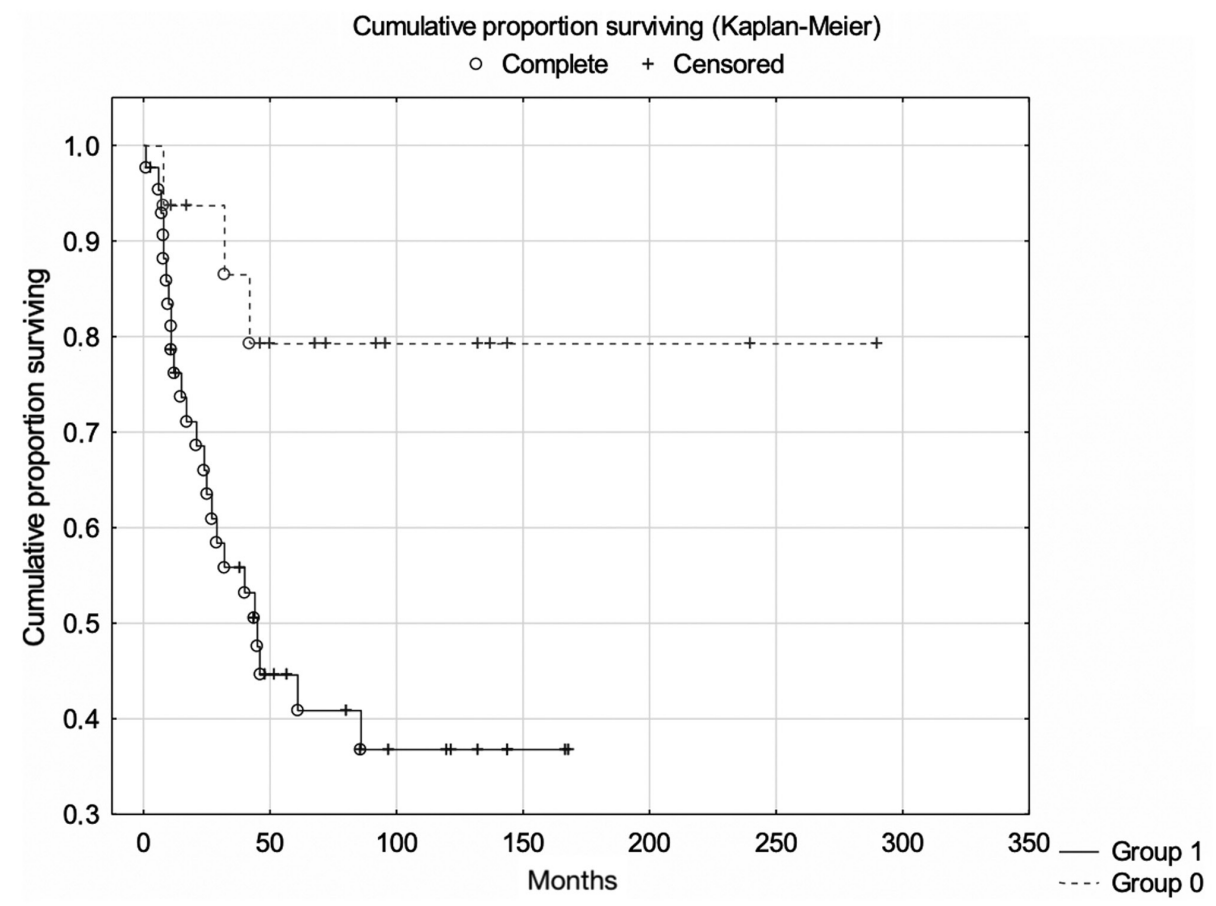

Figure 2. Cancer-specific survival rate vs. intensity of dyskerin staining. Group 0 , negative or $1+$ staining; group $1,2+$ or $3+$ staining. The difference in survival rate was highly significant between the two groups (log-rank test; $\mathrm{P}=0.009$ ).

The majority of cells (79\%) demonstrated a weak staining intensity of dyskerin, whereas $7 \%$ showed an moderate and $11 \%$ a strong staining intensity. Most of the staining was seen in nuclear bodies + nucleoplasm (57\%), not only in nucleoli.

Examination of dyskerin staining intensity in relation to clinical variables (Table II) revealed no association between the expression of dyskerin and FIGO stage at diagnosis (Pearson's $\left.\chi^{2} ; \mathrm{P}=0.509\right)$, nor with tumour localisation within the vagina $(\mathrm{P}=0.644)$. Although a high expression of dyskerin was more frequently observed in HPV-negative $(81 \%)$ compared with in HPV-positive tumour samples $(66 \%)$, this difference was not statistically significant $(\mathrm{P}=0.20)$ (data not shown). Examination of the relationship between the expression of dyskerin and histological type revealed that high expression was more frequently observed in basaloid and keratinising tumours $(87 \%)$ than in other histological types $(59 \%)$ $(\mathrm{P}=0.015$; data not shown). High expression of dyskerin was also significantly associated with poorly differentiated tumours $(\mathrm{P}=0.032)$ (Table II).

The primary cure rate was lower in patients with high expression of dyskerin in their tumour samples and those with low expression ( $81 \%$ vs. $94 \%$; $\mathrm{P}=0.241$ ); however, this change was not statistically significant. Similarly, overall recurrence among patients with high expression of dyskerin was $40 \%$, compared to $19 \%$ among those with low expression $(\mathrm{P}=0.134)$. Moreover, 13/14 recurrent tumours with distant metastasis demonstrated a high expression of dyskerin $(\mathrm{P}=0.001)$ (data not shown).

Examination of dyskerin staining intensity in relation to survival (Table II) revealed significant associations with disease progression and survival. Survival time was significantly shorter in patients with high expression of dyskerin compared with those with low expression (50 months vs.
92 months; $\mathrm{P}=0.017)$. In addition, $55.8 \%$ of patients with high expression of dyskerin had already died from PVC by the time this study was undertaken, while the corresponding figure for patients with low expression was $18.8 \%(\mathrm{P}=0.039)$.

High expression of dyskerin was associated with significantly lower 5-year cancer-specific survival rates (log-rank test; $\mathrm{P}=0.009$; Fig. 2). Expression of dyskerin remained a significant and independent prognostic factor after correction for age at diagnosis, tumour size, histological type and FIGO stage (Cox multivariate proportional regression analysis; $\mathrm{P}=0.035$; Table III).

Immunohistochemical analysis of WRAP53 $\beta$. As with dyskerin, WRAP53 $\beta$ was expressed in both nuclear bodies (12\%; likely Cajal bodies) and in nucleoplasm (16\%) (Fig. 3; Table IV). The majority of tumour samples showed expression of WRAP53 $\beta$, but no significant association was observed between the percentage of stained cells or staining intensity of WRAP53 $\beta$ and clinical variables or survival rates (data not shown).

\section{Discussion}

The present study demonstrates for the first time to the best of our knowledge that high expression of dyskerin, a protein involved in the modification of nuclear RNA and telomere elongation, is significantly associated with lower cancer-specific survival, as well as with lower overall survival in patients with PVC. One explanation is that dyskerin upregulation may lead to an increase in telomerase supramolecular complex formation, thus increasing the overall catalytic activity of telomerase (Fig. 4). Moreover, the higher dyskerin expression was significantly associated with poorly differentiated tumours. It was 
Table II. Correlation analysis of dyskerin expression and clinical variables and survival.

\begin{tabular}{|c|c|c|c|}
\hline Parameter & Group 0 & Group 1 & P-value \\
\hline Mean age at diagnosis, years ${ }^{\mathrm{a}}$ & 70 & 69 & 0.878 \\
\hline Mean tumour size, $\mathrm{mm}$ & 25 & 23 & 0.465 \\
\hline FIGO stage, n (\%) & & & 0.509 \\
\hline I & $7(43.8)$ & $12(28.0)$ & \\
\hline II & $6(37.5)$ & $19(44.2)$ & \\
\hline III & $2(12.5)$ & $4(9.3)$ & \\
\hline IV & $1(6.3)$ & $8(18.6)$ & \\
\hline Tumour localisation, n (\%) & & & 0.644 \\
\hline Upper & $5(31.2)$ & $12(27.9)$ & \\
\hline Middle & $4(25.0)$ & $7(16.3)$ & \\
\hline Lower & $4(25.0)$ & $14(32.6)$ & \\
\hline Entire vagina & $2(12.5)$ & $9(20.9)$ & \\
\hline Middle + lower & $0(0)$ & $1(2.3)$ & \\
\hline Urethra & $1(6.25)$ & $0(0)$ & \\
\hline Histological type, n (\%) & & & 0.226 \\
\hline Basaloid & $4(25)$ & $18(42)$ & \\
\hline Non-keratinizing & $9(56)$ & $13(30.2)$ & \\
\hline Keratinizing & $0(0)$ & $8(18.6)$ & \\
\hline Verrucous & $1(6.2)$ & $1(2.3)$ & \\
\hline Adenocarcinoma & $2(12.5)$ & $3(7)$ & \\
\hline Tumour grade, n (\%) & & & 0.032 \\
\hline Grade 1 & $2(12.5)$ & $6(14.3)$ & \\
\hline Grade 2 & $7(43.8)$ & $17(40.3)$ & \\
\hline Grade 3 & $3(18.8)$ & $18(42.9)$ & \\
\hline Unknown & $4(25)$ & $1(2.4)$ & \\
\hline HPV status, n (\%) & & & 0.360 \\
\hline Negative & $5(31.2)$ & $21(48.8)$ & \\
\hline Positive & $11(69)$ & $21(48.8)$ & \\
\hline Unknown & $0(0)$ & $1(2.3)$ & \\
\hline Primary cure rate, n (\%) & & & 0.241 \\
\hline Yes & $15(93.8)$ & $35(81.4)$ & \\
\hline No & $1(6.3)$ & $8(18.6)$ & \\
\hline Recurrence, n (\%) & & & 0.134 \\
\hline Yes & $3(18.9)$ & $17(39.5)$ & \\
\hline No & $13(81.3)$ & $26(60.5)$ & \\
\hline Mean time to recurrence, months & 29 & 19 & 0.511 \\
\hline Localisation of recurrent disease $(\mathrm{n}=20), \mathrm{n}(\%)$ & & & 0.619 \\
\hline Local & $2(66.7)$ & $3(17.6)$ & \\
\hline Regional & $0(0)$ & $1(5.9)$ & \\
\hline Distant metastasis & $1(33.3)$ & $9(52.9)$ & \\
\hline Local + regional + distant metastasis & $0(0)$ & $3(17.6)$ & \\
\hline Regional + distant metastasis & $0(0)$ & $1(0.6)$ & \\
\hline Survival status, n (\%) & & & 0.039 \\
\hline Alive & $5(31.3)$ & $8(18.6)$ & \\
\hline Death from disease & $3(18.8)$ & $24(55.8)$ & \\
\hline Death from intercurrent disease & $8(50.0)$ & $11(25.6)$ & \\
\hline Mean survival time, months & 50 & 92 & 0.017 \\
\hline
\end{tabular}

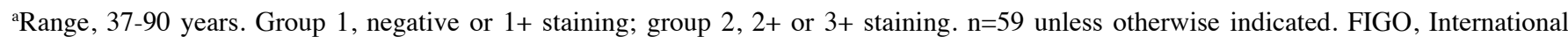
Federation of Gynecology and Obstetrics; HPV, human papillomavirus. 
Table III. Prognostic factors vs. cancer-specific survival rate.

\begin{tabular}{lcc}
\hline Factor & HR (95\% CI) & P-value \\
\hline FIGO stage (III-IV vs. I-II) & $1.669(0.659-4.224)$ & $\mathrm{ns}$ \\
Age at diagnosis (per year) & $1.020(0.980-1.062)$ & $\mathrm{ns}$ \\
Tumour size (per cm) & $1.099(0.738-1.636)$ & $\mathrm{ns}$ \\
Histology (squamous cell carcinoma vs adenocarcinoma) & $1.742(0.391-7.757)$ & $\mathrm{ns}$ \\
Dyskerin staining intensity (2-3 vs. 0-1) & $3.701(1.094-12.517)$ & 0.035
\end{tabular}

Cox multivariate proportional hazards analysis. HR, hazard ratio; CI, confidence interval; FIGO, International Federation of Gynecology and Obstetrics; ns, not significant.

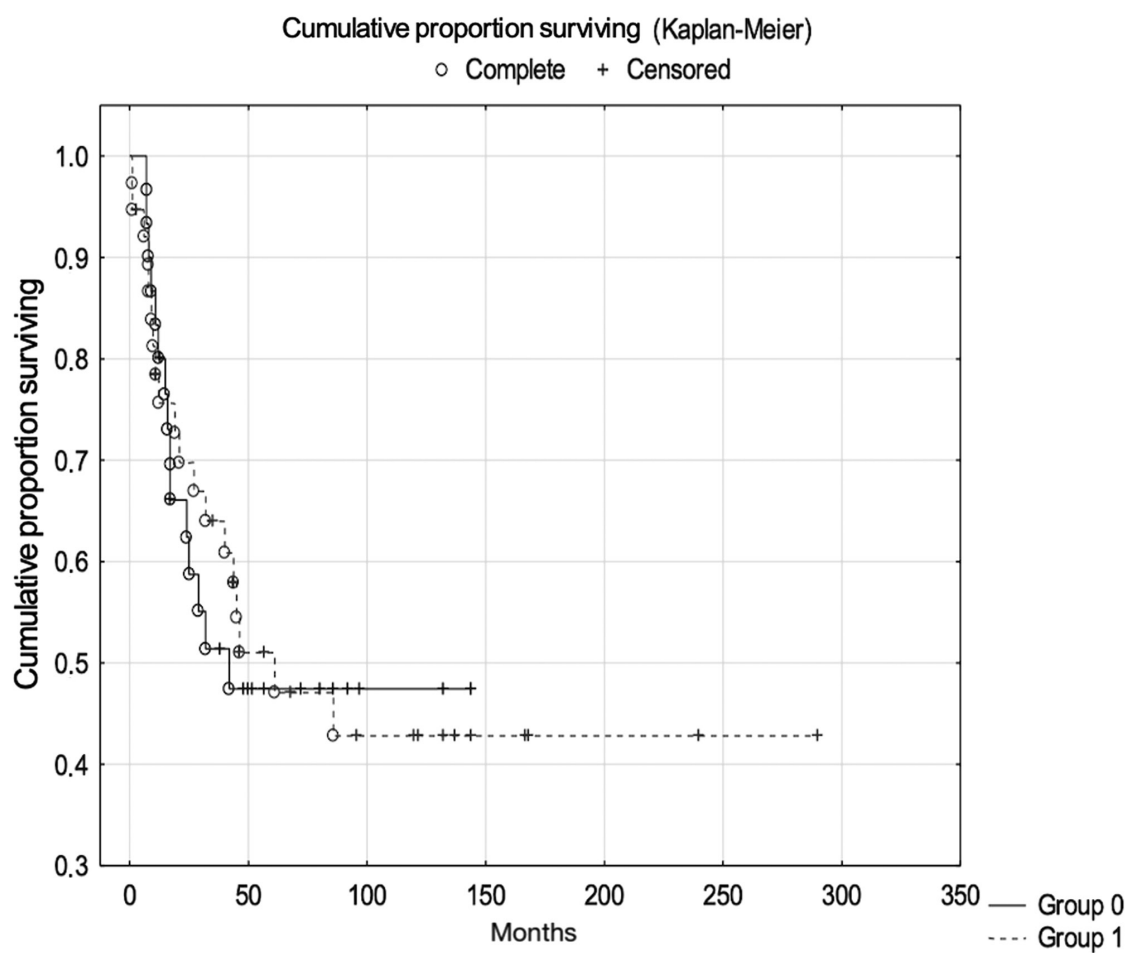

Figure 3. Cancer-specific survival rate vs. intensity of WRAP53 $\beta$ staining. Group 0 , negative or $1+$ staining; group $1,2+$ or $3+$ staining. There was no significant difference between the two groups (log-rank test; $\mathrm{P}=0.921)$. WRAP53 $\beta$, WD repeat containing antisense to TP53 $\beta$.

also associated with an increased risk of recurrent disease in terms of distant metastasis (Table II). In agreement with these findings, elevated dyskerin levels have been linked to progression and aggressiveness in several tumour types $(17,19,20,35)$ as well as to worse clinical outcomes in breast cancer (16), lung cancer (22), hepatocellular carcinoma (21), renal cell cancer (18) and neuroblastoma (23). Taken together, these findings suggest that dyskerin may be a useful prognostic marker for several types of cancer, including PVC.

A potential limitation of these findings is the limited number of patients included in this retrospective study. This reflects the low incidence of PVC (1) as well as the small population size in Sweden. However, given the non-parametric statistical model used in this study, the assumed risk of an underpowered study design is outweighed by the importance of performing exploratory studies on PVC. Indeed, previous knowledge on the biological factors of PVC has been based on similar sample sizes (2). In addition, due to the rarity of PVC, the present study was retrospective and based on archived, paraffin-embedded tumour samples instead of fresh-frozen material; the latter would have enabled complementary analyses. Nevertheless, future studies on a larger cohort with additional medical centres to confirm the present findings and to analyse the relationship between the expression of dyskerin and HPV infection in PVC, as well as in other gynaecological malignancies.

The mechanism by which dysregulation of dyskerin contributes to cancer development is debated, although it appears to be linked to both enhanced telomerase activity and protein biogenesis $(17,20)$. Non-small cell lung cancer provides one example of how high dyskerin expression is significantly associated with worse overall survival due to TERC stabilisation. Such stabilisation has been traced to the overexpression of dyskerin rather than to TERC gene amplification (22), which is in line with the idea that dyskerin can modify telomerase activity through the regulation of TERC levels, and independent of TERT expression (36). In the case of prostate cancer, 

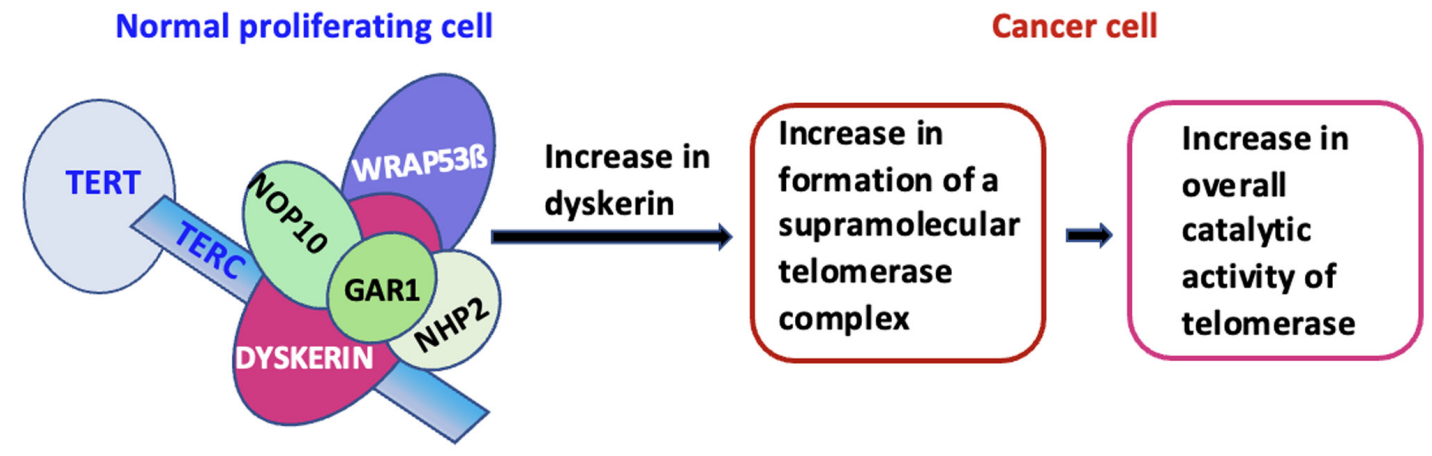

Figure 4. Schematic presentation of a putative role of dyskerin in primary vaginal cancer development. Upregulation of dyskerin leads to increase in telomerase supramolecular complex formation, thereby increasing the overall catalytic activity of telomerase. Dyskerin serves a structural scaffold of the telomerase complex. WRAP53 $\beta$, WD repeat containing antisense to TP53 $\beta$; TERT, telomerase reverse transcriptase; TERC, telomerase RNA complex.

Table IV. IHC analysis of WRAP53 $\beta$ staining intensity, fraction of stained cells and localisation pattern in tumour samples of patients with primary vaginal cancer.

\begin{tabular}{lc}
\hline IHC paramter & $\mathrm{n}(\%)$ \\
\hline WRAP53 $\beta$ IHC staining intensity & \\
0 & $8(8.5)$ \\
1 & $22(32.4)$ \\
2 & $20(29.4)$ \\
3 & $18(26.5)$ \\
WRAP53 $\beta$ IHC fraction of stained cells & \\
0 & $13(16.0)$ \\
1 & $32(39.5)$ \\
2 & $20(24.7)$ \\
3 & $3(3.7)$ \\
WRAP53 $\beta$ localisation ${ }^{\mathrm{a}}$ & \\
Nuclear bodies & $8(13.1)$ \\
Nucleoplasm & $11(18.0)$ \\
Nuclear bodies + nucleoplasm & $40(65.6)$ \\
Diffuse & $2(3.3)$ \\
\hline
\end{tabular}

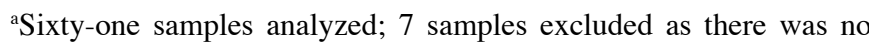
staining in the tumour cells. IHC, immunohistochemical; WRAP53 $\beta$, WD repeat containing antisense to TP53 $\beta$.

high expression of dyskerin mRNA is associated with more advanced clinical stage and recurrent disease. In this example, dysregulation of dyskerin is associated with enhanced protein biosynthesis rather than with telomerase activity (35). Indeed, loss of dyskerin function has been shown to reduce the amount of pseudouridinylated ribosomal RNA and thereby impair ribosome function and synthesis of proteins $(16,37)$.

Conversely, inactivating mutations of dyskerin cause dyskeratosis congenita, a rare genetic disease associated with a predisposition to cancer development (mainly haematological malignancies and head and neck cancer) (38). Similarly, dyskerin has also been shown to be downregulated in sporadic chronic lymphocytic leukaemia (39).

In light of the current study, upregulation of dyskerin might be associated with the poor prognosis of PVC due to enhancement of telomerase activity and/or altered protein synthesis. In an attempt to investigate the former, the expression of the WRAP53 $\beta$ protein, which is known to have a role in transporting dyskerin and the telomerase complex to telomeres as required for telomere elongation (14), was examined. The findings suggested that WRAP53 $\beta$ was expressed to varying degrees in the majority of PVC tumour samples, but this expression had no significant association with clinical parameters or patient survival. In PVC, the sub-cellular localisation of WRAP53 $\beta$ could not be linked to survival, in contrast with findings for both breast (27) and head and neck cancer (26). It can be concluded that the role of WRAP53 $\beta$ as a telomere transporter appears to be intact in PVC and that dyskerin upregulation could therefore result in enhanced telomere elongation. Upregulation of WRAP53 $\beta$ may also indicate its involvement in the DNA damage response, as suggested by Bergstrand et al (29).

Notably, the telomerase complex plays a crucial role in an important oncogenic pathway that stimulates the development and progression of HPV-associated cancer, which relates to the E6 oncogene of HPV that regulates TERT activity (40). Our finding of low expression of dyskerin in HPV-positive PVC tumour samples may suggest that dyskerin is indispensable to telomerase activity in these samples, since the E6 protein activates transcription of the human (h)TERT component of the telomerase complex (41). The association between gene expression linked to hTERT activity, including dyskerin, and malignant progression of HPV-induced cervical lesions, has been previously studied (42), although no correlation was observed between dyskerin expression at the protein level and the severity of precursor lesions. In the present study, dyskerin was detectable in nuclear bodies and/or in nucleoplasm, but not in the cytoplasm. Previous studies have detected dyskerin mainly in the nucleus, as well as in the cytoplasm of cervical precursor lesions (42), renal cell carcinoma (18) and hepatocellular carcinoma cells (21). One possible explanation for this discrepancy with our results may be the use of different dyskerin antibodies.

In summary, the present study demonstrates that upregulation of dyskerin is significantly associated with poor prognosis in PVC. This may be explained by the fact that an high expression of dyskerin may lead to increased telomerase supramolecular complex formation, thereby increasing the overall catalytic activity of telomerase. The future studies on PVC cell lines in vitro, using overexpression and downregulation of dyskerin with RNA sequencing analysis may clarify the functional 
consequences of dyskerin overexpression. In conclusion, the present findings point to dyskerin as a promising prognostic marker and as a potential putative therapeutic target in PVC.

\section{Acknowledgements}

Not applicable.

\section{Funding}

This study was supported by The Swedish Cancer Foundation (grant nos. 110544 and CAN2011/471), The Karolinska Institute Cancer Strategic Grants (grant no. 5888-05722), The Swedish Research Council (521-2008-2899), The Stockholm County Council (grant nos. 20130097 and 20160155), The Gustaf V Jubilee Fund (grant nos. 154022 and 151202) and The Centre of Clinical Research, Västmanland County Council (grant no. LTV-940144). The funding bodies had no role in the design of the study, the collection, analysis, or interpretation of data, or in the manuscript writing.

\section{Availability of data and materials}

The datasets used and/or analysed during the current study are available from the corresponding author on reasonable request.

\section{Authors' contributions}

CR, SA, MF, KH and GLL contributed to study design. GLL, LK and EK performed the immunohistochemical preparation. LK and MGK performed immunohistochemical analysis. BS and CR performed statistical analysis and interpretation of data was performed by CR, BS, and SA. DL helped with the writing of the manuscript and was involved in the planning of the study, formulating the hypothesis and study design. CR drafted the manuscript. BS and CR confirm the authenticity of all the raw data. All authors critically reviewed the manuscript. All authors have read and approved the final manuscript.

\section{Ethics approval and consent to participate}

The present study was approved by The Regional Ethical Review Board in Uppsala, Sweden (approval no. 2008/294), who did not request specific informed consent from patients. Patients were originally orally informed about the clinical research database. After 2003, they were also informed about tissue biobanking in accordance with the Swedish Biobank Act 2002:297. The study was performed in accordance with the Declaration of Helsinki.

\section{Patient consent for publication}

Not applicable.

\section{Competing interests}

The authors declare that they have no competing interests.

\section{Authors' information}

Dr Cecilia Ranhem's ORCID-ID is 0000-0002-3743-6217.

\section{References}

1. Sung H, Ferlay J, Siegel RL, Laversanne M, Soerjomataram I, Jemal A and Bray F: Global Cancer Statistics 2020: GLOBOCAN Estimates of Incidence and Mortality Worldwide for 36 Cancers in 185 Countries. CA Cancer J Clin 71: 209-249, 2021.

2. Hellman K, Silfversward C, Nilsson B, Hellstrom AC, Frankendal B and Pettersson F: Primary carcinoma of the vagina: factors influencing the age at diagnosis. The Radiumhemmet series 1956-96. Int J Gynecol Cancer 14: 491-501, 2004.

3. Hacker NF, Eifel PJ and van der Velden J: Cancer of the vagina. Int J Gynaecol Obstet 131 (Suppl 2): S84-S87, 2015.

4. Ghezelayagh T, Rauh-Hain JA and Growdon WB: Comparing mortality of vaginal sarcoma, squamous cell carcinoma, and adenocarcinoma in the surveillance, epidemiology, and end results database. Obstet Gynecol 125: 1353-1361, 2015.

5. Hiniker SM, Roux A, Murphy JD, Harris JP, Tran PT, Kapp DS and Kidd EA: Primary squamous cell carcinoma of the vagina: Prognostic factors, treatment patterns, and outcomes. Gynecol Oncol 131: 380-385, 2013.

6. Shah CA, Goff BA, Lowe K, Peters WA III and Li CI: Factors affecting risk of mortality in women with vaginal cancer. Obstet Gynecol 113: 1038-1045, 2009.

7. Gadducci A, Fabrini MG, Lanfredini $\mathrm{N}$ and Sergiampietri C: Squamous cell carcinoma of the vagina: Natural history, treatment modalities and prognostic factors. Crit Rev Oncol Hematol 93: 211-224, 2015.

8. Hellman K, Lundell M, Silfversward C, Nilsson B, Hellstrom AC and Frankendal B: Clinical and histopathologic factors related to prognosis in primary squamous cell carcinoma of the vagina. Int J Gynecol Cancer 16: 1201-1211, 2006.

9. Larsson GL, Helenius G, Andersson S, Sorbe B and Karlsson MG: Prognostic impact of human papilloma virus (HPV) genotyping and HPV-16 subtyping in vaginal carcinoma. Gynecol Oncol 129: 406-411, 2013.

10. Hellman K, Lindquist D, Ranhem C, Wilander E and Andersson S: Human papillomavirus, p16(INK4A), and Ki-67 in relation to clinicopathological variables and survival in primary carcinoma of the vagina. Br J Cancer 110: 1561-1570, 2014.

11. Hayflick L: The illusion of cell immortality. Br J Cancer 83: 841-846, 2000.

12. Blackburn EH: Switching and signaling at the telomere. Cell 106: 661-673, 2001.

13. Nguyen THD, Collins K and Nogales E: Telomerase structures and regulation: Shedding light on the chromosome end. Curr Opin Struct Biol 55: 185-193, 2019.

14. Venteicher AS, Abreu EB, Meng Z, McCann KE, Terns RM, Veenstra TD, Terns MP and Artandi SE: A human telomerase holoenzyme protein required for Cajal body localization and telomere synthesis. Science 323: 644-648, 2009.

15. Mahmoudi S, Henriksson S, Weibrecht I, Smith S, Söderberg O, Strömblad S, Wiman KG and Farnebo M: WRAP53 is essential for Cajal body formation and for targeting the survival of motor neuron complex to Cajal bodies. PLoS Biol 8: e1000521, 2010.

16. Montanaro L, Brigotti M, Clohessy J, Barbieri S, Ceccarelli C, Santini D, Taffurelli M, Calienni M, Teruya-Feldstein J, Trerè $\mathrm{D}$, et al: Dyskerin expression influences the level of ribosomal RNA pseudo-uridylation and telomerase RNA component in human breast cancer. J Pathol 210: 10-18, 2006.

17. Montanaro L, Calienni M, Bertoni S, Rocchi L, Sansone P, Storci G, Santini D, Ceccarelli C, Taffurelli M, Carnicelli D, et al: Novel dyskerin-mediated mechanism of p53 inactivation through defective mRNA translation. Cancer Res 70: 4767-4777, 2010.

18. Zhang M, Pan Y, Jiang R, Hou P, Shan H, Chen F, Jiang T, Bai J and Zheng J: DKC1 serves as a potential prognostic biomarker for human clear cell renal cell carcinoma and promotes its proliferation, migration and invasion via the NF- $\mathrm{KB}$ pathway. Oncol Rep 40: 968-978, 2018.

19. Bellodi C, Krasnykh O, Haynes N, Theodoropoulou M, Peng G, Montanaro L and Ruggero D: Loss of function of the tumor suppressor DKC1 perturbs p27 translation control and contributes to pituitary tumorigenesis. Cancer Res 70: 6026-6035, 2010.

20. Miao FA, Chu K, Chen HR, Zhang M, Shi PC, Bai J and You YP: Increased DKC1 expression in glioma and its significance in tumor cell proliferation, migration and invasion. Invest New Drugs 37: 1177-1186, 2019.

21. Liu B, Zhang J, Huang C and Liu H: Dyskerin overexpression in human hepatocellular carcinoma is associated with advanced clinical stage and poor patient prognosis. PLoS One 7: e43147, 2012. 
22. Penzo M, Ludovini V, Treré D, Siggillino A, Vannucci J, Bellezza G, Crinò L and Montanaro L: Dyskerin and TERC expression may condition survival in lung cancer patients. Oncotarget 6: 21755-21760, 2015.

23. O'Brien R, Tran SL, Maritz MF, Liu B, Kong CF, Purgato S, Yang C, Murray J, Russell AJ, Flemming CL, et al: MYC-Driven Neuroblastomas Are Addicted to a Telomerase-Independent Function of Dyskerin. Cancer Res 76: 3604-3617, 2016.

24. Mahmoudi S, Henriksson S, Corcoran M, Méndez-Vidal C, Wiman KG and Farnebo M: Wrap53, a natural p53 antisense transcript required for p53 induction upon DNA damage. Mol Cell 33: 462-471, 2009.

25. Henriksson S, Rassoolzadeh H, Hedström E, Coucoravas C, Julner A, Goldstein M, Imreh G, Zhivotovsky B, Kastan MB Helleday T, et al: The scaffold protein WRAP $53 \beta$ orchestrates the ubiquitin response critical for DNA double-strand break repair. Genes Dev 28: 2726-2738, 2014.

26. Garvin S, Tiefenböck K, Farnebo L, Thunell LK, Farnebo M and Roberg K: Nuclear expression of WRAP53 $\beta$ is associated with a positive response to radiotherapy and improved overall survival in patients with head and neck squamous cell carcinoma. Oral Oncol 51: 24-30, 2015.

27. Silwal-Pandit L, Russnes H, Borgen E, Skarpeteig V, Moen Vollan HK, Schlichting E, Kåresen R, Naume B, Børresen-Dale AL, Farnebo M, et al: The Sub-Cellular Localization of WRAP53 Has Prognostic Impact in Breast Cancer. PLoS One 10: e0139965, 2015.

28. Hedström E, Pederiva C, Farnebo J, Nodin B, Jirström K, Brennan DJ and Farnebo M: Downregulation of the cancer susceptibility protein WRAP53 $\beta$ in epithelial ovarian cancer leads to defective DNA repair and poor clinical outcome. Cell Death Dis 6: e1892, 2015.

29. Bergstrand S, O'Brien EM and Farnebo M: The Cajal Body Protein WRAP53 $\beta$ Prepares the Scene for Repair of DNA Double-Strand Breaks by Regulating Local Ubiquitination. Front Mol Biosci 6: 51, 2019.

30. Henriksson S and Farnebo M: On the road with WRAP53 $\beta$ : Guardian of Cajal bodies and genome integrity. Front Genet 6 : 91, 2015.

31. Zhang H, Wang DW, Adell G and Sun XF: WRAP53 is an independent prognostic factor in rectal cancer- a study of Swedish clinical trial of preoperative radiotherapy in rectal cancer patients. BMC Cancer 12: 294, 2012.

32. Rao X, Huang D, Sui X, Liu G, Song X, Xie J and Huang D: Overexpression of WRAP53 is associated with development and progression of esophageal squamous cell carcinoma. PLoS One 9: e91670, 2014.
33. Zhu Y, Ding L, Chen BF, Song JG and Yao YS: Oncogenic Activity of Wrap53 in Human Colorectal Cancer In Vitro and in Nude Mouse Xenografts. Med Sci Monit 24: 6129-6136, 2018.

34. Herrington SC and Ordi J: Chapter 34: Tumours of the vagina. In: WHO Classification of Tumours: Female genital tumours. 5th edition. Vol. 4. WHO Classification of Tumours Editorial Board (ed). IARC Press, Lyon, France, 2020.

35. Sieron P, Hader C, Hatina J, Engers R, Wlazlinski A, Müller M and Schulz WA: DKC1 overexpression associated with prostate cancer progression. Br J Cancer 101: 1410-1416, 2009.

36. Montanaro L, Calienni M, Ceccarelli C, Santini D, Taffurelli M, Pileri S, Treré D and Derenzini M: Relationship between dyskerin expression and telomerase activity in human breast cancer. Cell Oncol 30: 483-490, 2008.

37. Bellodi C, McMahon M, Contreras A, Juliano D, Kopmar N, Nakamura T, Maltby D, Burlingame A, Savage SA, Shimamura A, et al: H/ACA small RNA dysfunctions in disease reveal key roles for noncoding RNA modifications in hematopoietic stem cell differentiation. Cell Rep 3: 1493-1502, 2013.

38. Heiss NS, Knight SW, Vulliamy TJ, Klauck SM, Wiemann S, Mason PJ, Poustka A and Dokal I: X-linked dyskeratosis congenita is caused by mutations in a highly conserved gene with putative nucleolar functions. Nat Genet 19: 32-38, 1998.

39. PoncetD,Belleville A,t'kint de RoodenbekeC, Roborelde Climens A, Ben Simon E, Merle-Beral H, Callet-Bauchu E, Salles G, Sabatier L, Delic J, et al: Changes in the expression of telomere maintenance genes suggest global telomere dysfunction in B-chronic lymphocytic leukemia. Blood 111: 2388-2391, 2008.

40. Katzenellenbogen R: Telomerase Induction in HPV Infection and Oncogenesis. Viruses 9: E180, 2017.

41. Klingelhutz AJ, Foster SA and McDougall JK: Telomerase activation by the E6 gene product of human papillomavirus type 16 . Nature 380: 79-82, 1996.

42. Koskimaa HM, Kurvinen K, Costa S, Syrjänen K and Syrjänen S: Molecular markers implicating early malignant events in cervical carcinogenesis. Cancer Epidemiol Biomarkers Prev 19: 2003-2012, 2010.

(i) $(-)$ This work is licensed under a Creative Commons cc) International (CC BY-NC-ND 4.0) License. 